\title{
Post-Stroke Spasticity
}

\author{
Gerard E. Francisco, Jörg Wissel, Thomas Platz, \\ and Sheng Li
}

\section{Introduction}

Post-stroke spasticity (PSS) is a complication that contributes to limitations in performance of activities and community participation. It occurs in anywhere from 19\% (Sommerfeld et al. 2004) to 92\% (Malhotra et al. 2011) of stroke survivors. Its prevalence may be as high as $38 \%$ in the first year following a stroke (Watkins et al. 2002). Estimates of incidence and prevalence vary widely perhaps due differences in the definition and clinical measurement of spasticity in varying severity and chronicity of stroke. As well, based on a prospective, observational study, Wissel et al. (2010) found a prevalence of spasticity in $24.5 \%$ at 6 days, $26.7 \% 6$ weeks, and $21.7 \%$ (18 of 83 survivors at 16 weeks). The same group also found that in $98 \%$ of

\footnotetext{
G. E. Francisco $(\bowtie) \cdot$ S. Li

Department of Physical Medicine and Rehabilitation, The University of Texas Health Science Center at Houston McGovern Medical School and TIRR Memorial Hermann Hospital, Houston, TX, USA e-mail: gerard.e.francisco@uth.tmc.edu; Sheng.Li@uth.tmc.edu

J. Wissel

Department of Neurology, Neurorehabilitation and Physical Therapy, VIVANTES Hospital Spandau, Berlin, Germany

University of Potsdam, Health Science, Health Campus Brandenburg, Potsdam, Germany e-mail: Joerg.Wissel@vivantes.de

\section{T. Platz}

Institute for Neurorehabilitation and Evidence-based Practice ("An-Institute", University of Greifswald), BDH-Klinik Greifswald, Greifswald, Germany

Neurorehabilitation Research Group, University Medical Centre Greifswald (UMG), Greifswald, Germany

Special Interest Group Clinical Pathways, World Federation Neurorehabilitation (WFNR), North Shields, UK

e-mail: T.Platz@bdh-klinik-greifswald.de
} 
subjects with PSS, velocity-dependent increase in muscle tone in one joint was evident at about 6 weeks post-stroke.

Beside identified early clinical signs that predict PSS (velocity-dependent increase in muscle tone in two or more joints, severe paresis and loss of function resulting in severe disability/loss of ADL), recent studies showed that brain lesions involving the basal ganglia, thalamus, insula, and white matter tracts especially the internal capsule, corona radiata, external capsule, and superior longitudinal fasciculus are also predictive for PSS when lesion load is compromising the corticospinal tract (Wissel et al. 2015; Lee et al. 2019).

There is a heightened awareness of the need to manage spasticity because not only can it limit limb movement and overall mobility but also can predispose to other complications, such as joint contractures and pain, which further magnify motor weakness and functional limitations. In this chapter, current evidence will be assessed in the following key clinical areas:

- Problem identification and clinical assessment.

- Treatment goal setting.

- Pharmacological and surgical treatment.

\section{Methods Used for Evidence Synthesis and Practice Recommendations}

A comprehensive literature search was performed on 30 July, 2017 on PubMed (MEDLINE database) for guidelines, systematic reviews and meta-analyses (SR), and randomized controlled studies (RCT) published in the last 5 years on the topic of spasticity treatment across etiologies. This was performed in conjunction with preparations for the German Neurology Society S2k (consensus) guideline on the "Treatment of spasticity" (Platz et al. 2019). Search terms for SR and RCT were: (1) (Search (meta analysis [Publication Type] OR meta analysis [Title/Abstract] OR meta analysis [MeSH Terms] OR review[Publication Type] OR search*[Title/ Abstract]) AND (spast*)); (2) (Search ((clinical[Title/Abstract] AND trial[Title/ Abstract]) OR clinical trials as topic[MeSH Terms] OR clinical trial[Publication Type] OR random*[Title/Abstract] OR random allocation[MeSH Terms] OR therapeutic use[MeSH Subheading]) AND (spast* OR ton*)) AND ("last 5 years"[PDat]). The search initially yielded 297 references, and after eliminating duplicates, 215 references remained, of which 28 were directly relevant for the preparation of said guideline. The vast majority of the 28 manuscripts were systematic reviews.

Additionally, the S2e (evidence-based) German Society for Neurorehabilitation (DGNR) guideline on "Treatment of Spasticity after Stroke" (Winter and Wissel 2013) was referenced and all essential information included. For the DGNR guideline (Winter and Wissel 2013), 172 references (164 original papers, 8 reviews) were evaluated, of which 111 served as a basis for the guideline, which included non-experimental studies (e.g., case series and cohort studies). In addition, further studies and reviews that were known to the authors and relevant for the healthcare question addressed were included.

The recommendations given below are based on the evidence as presented below. We categorized the level of evidence used for recommendations according to the 
Oxford Centre for Evidence-Based Medicine Levels of Evidence (CEBM 2009). Further, the quality of this evidence has been grouped into four categories according to "GRADE" ("Grades of Recommendation, Assessment, Development and Evaluation") (Owens et al. 2010):

- High quality: further research is unlikely to affect our confidence in the estimation of the (therapeutic) effect.

- Medium quality: further research is likely to affect our confidence in the estimation of the (therapeutic) effect and may alter the estimate.

- Low quality: further research will most likely influence our confidence in the estimation of the (therapeutic) effect and will probably change the estimate.

- Very low quality: any estimation of the (therapy) effect or prognosis is very uncertain.

The grading of the recommendations according to GRADE (Schünemann et al. 2013) corresponds to the categories "ought to" (A) (strong recommendation), "should" (B) (weak recommendation). As a third category had been introduced "can" (0) (option) (Platz 2017). Recommendation category A is granted for clinically effective interventions with high-quality evidence support (and when mandatory, e.g., for ethical reasons); with medium-quality evidence category B, and with low- or very low-quality evidence category 0 can be appropriate. Reasons to upgrade or downgrade are given: $\mathrm{A}+$ or $\mathrm{B}+$ denote a strong or weak recommendation in favor on an intervention and $\mathrm{A}-$ or $\mathrm{B}-$ against its use.

\section{$3 \quad$ Problem Identification and Clinical Assessment}

When assessing clinical problems associate with post-stroke spasticity, it is very important to keep in mind that spasticity defined as muscle overactivity as a positive sign of the upper motor neuron syndrome (UMNS) is only one component. The problems may be caused by other motor impairments, such as weakness, and/or disordered motor control. For example, patients may complain of abnormal joint positions, or that a limb could not be moved, or the resultant functional limitations, such as the inability to release a grasped object or difficulty with walking due to an inverted foot. In the majority of post-stroke patients, these problems are a combination of the above motor impairments. Thus, it is important to obtain a thorough, yet focused, history to identify clinical problems, to guide the examination, and to formulate treatment goals and plans. A systematic approach to history-taking and clinical assessment of spasticity that can be modified to suit different clinical scenarios is proposed in Tables 1 and 2.

With respect to the distribution of the positive signs of the UMNS identified as involuntary muscle overactivity (velocity-depended increase in muscle tone, spasms, clonus, spastic dystonia, and co-contracting muscle activation) spasticity could be classified as focal (one joint/functional region, e.g., the wrist and fingers), multifocal (minimal two or more joints, e.g., wrist, fingers, and ankle), segmental (adjacent joints in one or more limbs, e.g., toes, ankle, knee, and hip in one or two limbs, paraspastic: both lower limbs), hemispastic (arm and leg on one body side), 
Table 1 Some important historical points in spasticity assessment

Is the limb tight all the time or only at certain times?

Does a particular position or movement trigger tightness?

Is the tightness related to spasms?

Does the tightness cause pain?

Have there been episodes of skin compromise due to tightness or spasm?

Does the tightness result in difficulty with cleaning?

Does the tightness result in difficulty donning splints?

Does the tightness limit ability to move limbs, reach for objects, and use the hands?

Does the tightness of the lower limbs result in problems with transferring form one surface to another or with walking?

What treatments for muscle tightness have been tried previously and their outcome?

What are the current medications?

Was there a recent increase in tightness (that may warrant further diagnostic testing to rule out a new neurologic problem)?

Any recent medical problems?

From Francisco GE and Li S. Clinical assessment and management of spasticity and contractures in traumatic brain injury. Chapter 8 In Neurological Rehabilitation Spasticity and Contractures in clinical practice and research. Pandyan AD, Hermens H, Conway B (2018). https://doi. org/10.1201.9781315374369

Table 2 Practical clinical examination sequence

\begin{tabular}{l|l|l}
\hline $\begin{array}{l}\text { Examination } \\
\text { phase }\end{array}$ & What to look for & What can be gleaned \\
\hline Observation & $\begin{array}{l}\text { Observe limb posture at rest and how } \\
\text { they change with position }\end{array}$ & $\begin{array}{l}\text { Abnormal posture at rest- } \\
\text { Sustained muscle contraction } \\
\text { (dystonia), contracture } \\
\text { Position-dependent postural } \\
\text { changes-dynamic tone }\end{array}$ \\
\hline Active $^{\text {a }}$ & $\begin{array}{l}\text { How limbs move and how much active } \\
\text { range is available } \\
\text { Gait characteristics and associated upper } \\
\text { limb and trunk postural abnormalities }\end{array}$ & $\begin{array}{l}\text { Functional strength, coordination, } \\
\text { spastic co-contraction, contractures, } \\
\text { presence of other movement } \\
\text { disorders, synkinesis, or associated } \\
\text { reactions } \\
\text { Pain and discomfort during } \\
\text { voluntary movements }\end{array}$ \\
\hline Passive & $\begin{array}{l}\text { Passive range of motion, strength, muscle } \\
\text { tone, velocity-dependent "angle of } \\
\text { catch," clonus }\end{array}$ & $\begin{array}{l}\text { Spasticity } \\
\text { Rigidity } \\
\text { Contracture } \\
\text { Clonus } \\
\text { Pain and discomfort during passive } \\
\text { stretch }\end{array}$ \\
\hline $\begin{array}{l}\text { Functional } \\
\text { activities }\end{array}$ & $\begin{array}{l}\text { Performance of specific tests and tasks } \\
\text { (both formal tests, such as Frenchay and } \\
\text { improvised tasks such as demonstrating } \\
\text { ability to pick up a bottle of water and } \\
\text { pour its contents to a cup) }\end{array}$ & $\begin{array}{l}\text { Impact of multiple impairments } \\
\text { (e.g., spasticity, weakness) on } \\
\text { performance }\end{array}$ \\
\hline
\end{tabular}

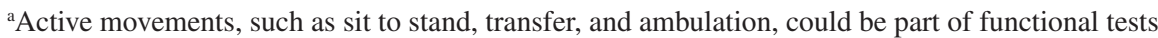


or generalized (more than two limbs involved, e.g., both legs and additional focal or segmental distribution of spasticity in arms, jaw, and trunk (Wissel et al. 2009)).

Spasticity assessment typically consists of a combination of quantitative and qualitative measures. Clinically, the Ashworth scale (AS), the modified Ashworth scale (MAS), and the Tardieu scale (TS) are most commonly used (Tables 3 and 4). Standardization of performance and scoring and summary scores across muscles groups were developed for the AS and resulted in high internal consistency, interrater, and test-retest reliability of a summary rating scale (REsistance to PASsive movement scale, REPAS) (Platz et al. 2008). A recent meta-analysis study reports satisfactory scores of inter- and intra-rater reliability in MAS, particularly when used for the upper extremity (Meseguer-Henarejos et al. 2018). The TS has advantages over the MAS because not only it quantifies the muscles' reaction to passive stretch but also it controls for the velocity of the stretch and measures the angle at which the catch, or clonus activity, occurs (Haugh et al. 2006); here, however, issues of reliability still have to be solved for the clinical user (Ansari et al. 2013; Li et al. 2014; Banky et al. 2019). In patients with severe brain injury, the MTS was shown to provide higher test- retest and inter-rater reliability compared to MAS (Mehrholz et al. 2005). This is supported by other investigations (Paulis et al. 2011; Singh et al.

Table 3 Ashworth scale and modified Ashworth scale

\begin{tabular}{l|l}
\hline \multicolumn{2}{l}{ Ashworth scale } \\
\hline 0 & No increase in tone \\
\hline 1 & Slight increase in tone giving a catch when the limb was moved in flexion or extension \\
\hline 2 & More marked increase in tone but limb easily moved \\
\hline 3 & Considerable increase in tone, passive movement difficult \\
\hline 4 & Limb rigid in flexion or extension \\
\hline Modified Ashworth scale \\
\hline 1 & No increase in muscle tone \\
\hline $1+$ & $\begin{array}{l}\text { Slightly increase in tone, manifested by a catch, followed by minimal resistance } \\
\text { throughout the remainder (less than half) of the ROM (catch in the first half of ROM) }\end{array}$ \\
\hline 2 & Marked increase in tone through most of the ROM, still easily moved \\
\hline 3 & Considerable increase in tone, passive movement difficult \\
\hline 4 & Affected part(s) rigid in flexion or extension \\
\hline
\end{tabular}

Table 4 Tardieu scale

\begin{tabular}{|c|}
\hline Quality of muscle reaction \\
\hline 0 . No resistance \\
\hline 1. Slight resistance \\
\hline 2. Catch followed by a release \\
\hline 3. Fatigable clonus $(<10 \mathrm{~s})$ \\
\hline 4. Infatigable clonus (>10 s) \\
\hline Angle of muscle reaction at different velocities of stretch \\
\hline V1. As slow as possible \\
\hline V2. Speed of limb galling under gravity \\
\hline V3. As fast as possible \\
\hline
\end{tabular}


Table 5 The SpasticityAssociated Arm Pain Scale (SAAPS) for adults with post-stroke upper limb spasticity: passive range of motion items and ratings

\begin{tabular}{l|l}
\hline Item & Description \\
\hline 1 & Paretic shoulder abduction with elbow flexed to $90^{\circ}$ \\
\hline 2 & $\begin{array}{l}\text { Paretic shoulder external rotation with elbow flexed } \\
\text { to } 90\end{array}$ \\
\hline 3 & Elbow stretching/extension \\
\hline 4 & Wrist stretching \\
\hline 5 & Finger stretching \\
\hline Score & Observation \\
\hline 0 & No pain \\
\hline 1 & $\begin{array}{l}\text { Pain on repeated movement (maximum of five } \\
\text { repetitions) }\end{array}$ \\
\hline 2 & Pain on end-range movement \\
\hline 3 & Immediate pain on movement \\
\hline
\end{tabular}

Verbal and/or physiological responses (pain responses) are rated on a four-point Likert-type scale as 0 ("no pain"), 1 ("pain on repeated movement" (maximum of five repetitions)), 2 ("pain on end-range movement"), or 3 ("immediate pain on movement"). The SAAPS sum score ranges from 0 to 15 points, whereas 0 is the lowest and 15 is the highest pain intensity during passive range of motion in moderate speed of movement

2011). Caution regarding reliability, however, was raised in at least two investigations (Ansari et al. 2008, 2013; Li et al. 2014).

To assess pain associated with arm spasticity following stroke a valid and reliable instrument, the Spasticity-Associated Arm Pain Scale (SAAPS), for adults with post-stroke upper limb spasticity was developed (see Table 5; (Fheodoroff et al. 2017)). Most studies published on spasticity-related pain have used nonspecific pain-assessment scales, e.g., the 11-point box scale (NRS) or visual analog scale (VAS). However, such scales have not been validated in patients with spasticityrelated arm pain and may lack the sensitivity to detect change and may also be unsuitable for use in patients in nursing care homes, many of whom have severe cognitive impairment or late-stage dementia (Lichtner et al. 2014; Tyson and Brown 2014). To address these shortcomings when documenting or managing pain in patients with arm spasticity following stroke, the SAAPS was developed and evaluated and showed its sensitivity for detecting and documenting pain and its reduction following BoNT-A treatment in pain associated with arm spasticity following stroke (Fheodoroff et al. 2017).

While it is true that quantitative measures are desirable because of their inherent objectivity and reliability, they may not be practical and may discourage clinicians from assessing and managing spasticity. Quantitative assessment includes biomechanical and electrophysiological assessments. Biomechanical assessment utilizes the concept of velocity-dependent increase in resistance and has advantages of the ability to differentiate neural and nonneural component of spastic hypertonia, thus spasticity vs. contracture (Sinkjaer and Magnussen 1994; Kamper et al. 2003; Li et al. 2006). The measurement of electromyographic activity is able to determine the threshold of stretch reflex, i.e., a physiological evidence of the onset of spasticity (Levin and Feldman 1994; Calota et al. 2008). Advances in quantitative ultrasound 
imaging have shown some promising pilot data to quantitatively assess spastic muscles (Wu et al. 2017; Gao et al. 2018). Ideal spasticity assessment should include biomechanical and electrophysiological tests, but many of the devices are not available to a typical clinician, and the time needed to perform them properly may impose excessive demands in a busy practice.

Recommendations: It is important to keep in mind that spasticity could be one component of motor impairments and clinical problems following stroke and may contribute to reduction in activities of daily living and quality of life in about $10-12 \%$ of chronic stroke survivors. Spasticity should be classified according to the topical distribution as focal, multifocal, segmental, hemispastic, paraspastic, or as generalized spasticity. It should primarily be assessed and documented by standardized validated clinical assessment scales such as the AS or MAS (level of evidence $2 \mathrm{a}$, quality of evidence moderate, $\mathrm{B}+$ ) or TS (level of evidence $2 \mathrm{~b}$, quality of evidence low, 0).

\section{$4 \quad$ Treatment Goal Setting}

\subsection{Goal Setting}

The mere presence of spasticity is not always a reason to initiate treatment. Varying in its severity, spasticity is usually addressed when it presents with marked limitation in range of motion due to abnormally increased muscle tone. Consideration, however, should be given to managing less-than-severe spasticity if it has profound impact on comfort and functioning. An example is a person with pes equinovarus deformity whose ankle plantar flexors and invertors merit only a modified Ashworth Scale score of $1+$ at rest, but causes discomfort during walking. Spasticity intervention ought to be considered not only based on severity but also on functional significance and impact on well-being. Thus, the first important step in managing spasticity, and right after problem identification, is laying out the rationale for treatment by identifying goals. Goals, especially patient-centered ones, are a basis for collaboration by patients and clinicians. Together (patient/care giver and clinician), they agree on the desired treatment outcome, which will be based on the extent to which goals were achieved. To assist in developing goals, a useful matrix, SMARTER, can be used (Francisco and Li 2015). This mnemonic stands for goals that are:

- S: Specific (well-defined and targets a specific problem to be addressed).

- M: Measurable (either quantitatively, as for technical goals, or qualitatively as for symptom-directed goals); Meaningful (achievement of goal should be beneficial to the patient or caregiver).

- A: Agreed upon (the patient or caregiver and clinician work toward a common end).

- R: Realistic (will the patient's potential for improvement and available resources support achievement of treatment goal?)

- T: Time-bound (achievement of goal should be within a reasonable amount of time). 
- E: Evaluated (at pre-determined points in time, goal achievement and progress in doing so should be performed to determine effectiveness of intervention).

- R: Revised (based on evaluation of goal achievement, new treatment goals may be identified or prior ones revised).

A recent national, multicenter study (Ashford et al. 2016) suggested that goals can be classified into two domains, namely, symptoms and impairment, and activities/function. The former includes pain/discomfort, involuntary movements, and range of movement/contracture prevention. Within the second domain are passive function (e.g., ease of caring for affected limb), active function (using affected limbs to perform tasks), and mobility.

\subsection{Goal Attainment Scaling}

The most commonly used measure to document treatment goals is goal attainment scaling (GAS), which was originally used in other healthcare settings (Kiresuk and Sherman 1968; Rockwood et al. 1997). GAS tracks the extent to which pre-identified patient-specific treatment goals are achieved using an ordinal scale during the course of treatment. The scoring method is standardized to promote consistent repeatability as the patient and clinician review the progress of goal achievement. In addition, early studies comparing GAS with more traditional outcome measures suggest that GAS is more sensitive. Active patient engagement through GAS may have beneficial impact on achievement of outcomes. Emerging evidence demonstrate that when patients are involved in goal setting, they are more likely to achieve their goals (Turner-Stokes et al. 2015). GAS also shows promise as a means of conveying caregiver burden as it is sensitive in highlighting priority outcomes by patients and caregivers (Turner-Stokes et al. 2010).

Recommendation: An important component of assessment and management decision-making is arriving at treatment goals. Identifying goals that are mutually agreed upon by the patient, caregiver, and clinician a priori should be an important step in spasticity treatment decision-making (level of evidence $2 \mathrm{~b}$, quality of evidence moderate, $\mathrm{B}+$ ). In this context, GAS is a useful tool in negotiating goals, highlighting priority outcomes by patients and caregivers, and tracking their achievement. GAS also encourages patient engagement in goal setting, which has been shown to have a positive relation to achieving the said goals.

\section{$5 \quad$ Pharmacological and Surgical Treatment}

\subsection{Systemic Medications}

Various oral medications with different mechanisms of action have been used to treat spasticity. The most commonly used medications include baclofen, tizanidine, dantrolene and benzodiazepines. Overall, these medications have marginal effect on 
focal spasticity (Simpson et al. 2009) and marginal to moderate effects on reducing segmental and generalized spasticity (Montane et al. 2004). In a systematic review on contemporary pharmacologic treatments for spasticity of the upper limb after stroke. Olvey et al. (2010) only found one study with a systemic drug (tizanidine) that reported significant reductions in upper limb spasticity after 16 weeks of treatment compared to placebo.

In a Cochrane review on pharmacological interventions other than botulinum toxin for spasticity after stroke, Lindsay et al. (2016) found seven RCTs with a total of 403 participants. Only two of them assessed a systemic drug versus placebo and only one with tizanidine showed significant results. Those two studies included 160 patients and in the meta-analysis of those the antispastic effect of the oral drugs on spasticity showed no significant effects (MAS, Odds Ratio for Response 1.66, 95\% KI 0.21-13.07; n.s.). On the other hand, the authors identified a significant risk of adverse events per participant occurring in the treatment group versus placebo group (risk ratio (RR) $1.65,95 \%$ CI 1.12 to 2.42 ; 160 participants; $I^{2}=0 \%$ ).

The common adverse effects of oral drugs include dose-dependent adverse effects, such as drowsiness and tiredness. Therapeutic efficacy of these medications is only supported by a few placebo-controlled trials with inadequate sample size and lack of functional outcome measurements (Francisco and McGuire 2012; Winstein et al. 2016). Furthermore, likely attributed to these adverse effects or a reflection of limited efficacy, oral medications have poor adherence (Halpern et al. 2013). In general, it is recommended to limit the use of these oral medications for spasticity management (Francisco and McGuire 2012; Winstein et al. 2016). On the other hand, these oral medications as single drug or combination may be a cost-effective treatment option for those who can achieve adequate spasticity management without intolerable adverse effects.

Baclofen, a gamma amino butyric acid (GABA)-B agonist, is a potent inhibitory neurotransmitter. Baclofen has been shown dose-dependent reduction of spasticity and spasms, but has side effects of drowsiness and weakness. Abrupt discontinuation of baclofen may result in a withdrawal syndrome, characterized by rebound spasticity, hallucinations, and seizures (Medaer et al. 1991; Meythaler et al. 2004). Similar to baclofen, benzodiazepines modulate GABAergic transmission by binding GABA-A receptors. Similarly, abrupt discontinuation of benzodiazepines may result in a withdrawal syndrome. Concerns for its side effects, mainly drowsiness, sedation, reduced attention and memory impairment, and the potential for physiological dependence have limited the use of benzodiazepines as first-line treatment for spasticity. The use in post-stroke spasticity management is discouraged and might be reserved to situations when spasticity is accompanied by other conditions that are also amenable to benzodiazepine therapy, such as seizures, anxiety, insomnia, spasms, and other movement disorders (Medaer et al. 1991; Meythaler et al. 2004).

Tizanidine, a central alpha2-adrenergic receptor agonist, reduces spasticity and clonus via inhibiting the facilitatory ceruleospinal tracts and the release of excitatory neurotransmitters from spinal interneurons (Stevenson and Jarrett 2006). In addition to the typical side effects of oral spasmolytics, hepatotoxicity may also 
occur. Thus, monitoring liver function is important, especially in those patients who concomitantly take hepatically cleared drugs.

Unlike baclofen and tizanidine, dantrolene works directly on skeletal muscle by inhibiting the release of calcium from the sarcoplasmic reticulum during excitationcontraction coupling (Krause et al. 2004). Although it is peripherally acting, dantrolene has also been associated with side effects that appear to be centrally mediated, such as drowsiness, dizziness, fatigue, and weakness, perhaps through alteration of neuronal calcium homeostasis (Flewellen et al. 1983; Katrak et al. 1992). Due to its potential for hepatotoxicity, regular monitoring of liver function is recommended.

Beside baclofen, tizanidine, and dantrolene, other agents have reported to have some effects, including tolperison (Stamenova et al. 2005), gabapentin, clonidine, nabilone (Wissel et al. 2006), and cannabinoids (Whiting et al. 2015).

Summing up the results from studies on oral drugs in PSS, the evidence published from RCTs showed significant risk of side effects and no sufficient data to confirm that systemic antispastic drugs are effective in treating PSS.

Recommendations: Oral systemic medications can be used for segmental and generalized spasticity, but may be associated with dose-dependent adverse effects (level of evidence $2 b$, quality of evidence low, 0 ). Selection of type of oral medication depends on individual circumstances and may include combinations. These medications should be titrated slowly, and both clinical benefits and unwanted effects need to be monitored (level of evidence 1a, quality of evidence low, B+ [clinical reasoning]).

\subsection{Botulinum Toxin Treatment}

Botulinum toxin Type A (BoNT-A) is widely regarded as the treatment of choice for the medical management of focal and multi-focal signs and symptoms of the UMNS including but not limited to hypertonia, spastic dystonia, clonus, and spasms. Systematic studies have also demonstrated improvement in so-called passive function like reducing spasticity-associated pain, hygiene and passive movement of involved limbs, and reduction of mal-positioning of limbs (Brashear et al. 2002a, b) (level of evidence 1b). Demonstration of active functional gains in terms of enhancement of active limb movements in the upper limbs (e.g., reaching or grip and relieve movements with the hand) and increased mobility (e.g., gait speed and gait endurance) has proven to be difficult. But systematic reviews of the outcome of BoNT treatment in spastic upper limbs (Foley et al. 2013; Dong et al. 2017) (level of evidence 1a) and a randomized, controlled, trial in subjects with chronic PSS and traumatic brain injury-related spastic upper limb (Gracies et al. 2015) (level of evidence 1b) reported statistically significant functional improvements in active function of the upper limb.

Controlled studies in the post-acute phase of stroke rehabilitation (less than 3 months following stroke) showed that BoNT-A injected before spasticity became moderate or severe result in improvements in impairment and passive function and reduced occurrence of muscle and/or tendon shortening in long finger flexors (Hesse 
et al. 2012). The therapeutic outcomes also appeared to be more pronounced and longer lasting (Rosales et al. 2012; Fietzek et al. 2014) (level of evidence 1b). Thus, early BoNT-A intervention seems to have the potential to modify the natural evolution of PSS while a resent systematic review did not indicate higher functional gains or effects on disability (Rosales et al. 2016).

\subsubsection{Treatment Outcomes: Upper Limbs}

Numerous controlled studies and multiple meta-analyses show a dose-dependent effective reduction of spastic muscle tone, improvement in passive range of motion (PROM) and passive function (measured with the Disability Assessment Scale, DAS) as well as a reduction in carer burden while handling affected limbs, both by single and repeated intramuscular injections of BoNT-A (abobotulinumtoxinA, incobotulinumtoxinA, and onabotulinumtoxinA) in upper limb with increased spastic hypertonia in the chronic stage after stroke and other etiologies ((van Kuijk et al. 2002; Turkel et al. 2006), level of evidence 1a; (Simpson et al. 2008, 2016), level of evidence 1a; (Gracies et al. 2015), level of evidence 1b; (Dong et al. 2017), level of evidence 1a)). In severely affected arms, BoNT-A application supports self-care activities and integration of the spastic arm in everyday life by improving passive function (Baker and Pereira 2015) (level of evidence 1a). Evidence quality for the 11 studies for arm spasticity in a meta-analysis was moderate (GRADE). Significant results of BoNT-A therapy were observed for 4 to 12 weeks post-injection (SMD $0.80,95 \%$ CI 0.55 to $1.06, P<0.0001$ ) and continued for up to 6 months (SMD $0.48,95 \%$ CI 0.34 to $0.62, P<0.0001$ ). Randomized trials included in the systematic review showed a reduced incidence of shortening of finger muscles with improved hand hygiene (Hesse et al. 2012; Rosales et al. 2012) (level of evidence $1 b)$.

In the subacute phase ( $<3$ months) after stroke following BoNT-A use, forearm and leg spasticity significant reductions in velocity-dependent tone increase over more than 3 months could be documented in a systematic review with meta-analysis (3 studies), whereas no greater functional gains and no increase in side effects were shown (Rosales et al. 2016) (level of evidence 1a).

BoNT-A injections may improve active function in some of the patients with arm spasticity (Foley et al. 2013; Gracies et al. 2015; Baker and Pereira 2016, level of evidence 1a). Evidence quality for the six studies for effects on arm activities in a meta-analysis was low to very low (according to GRADE). A small significant result of BoNT-A therapy was documented 4 to 12 weeks post-injection (SMD 0.32, 95\% CI 0.01 to $0.62, P=0.04$ ) and only for the Action Research Arm Test (ARAT) persisted for up to 6 months (MD 1.87, 95\% CI 0.53 to 3.21, $P=0.006$ ). In a single randomized controlled trial of abobotulinumtoxin $\mathrm{A}$, an increase in active finger and wrist extension with BoNT-A injections into the finger flexors was found and supported the prior statement (Gracies et al. 2015), (level of evidence 1b). For treatment of focal spasticity after stroke, BoNT-A is superior to oral antispastic medication (tizanidine) in terms of both efficacy, measured as reduction in muscle tone with the Modified Ashworth Scale, and adverse events (Simpson et al. 2009) (level of evidence $1 b)$. 
Recommendations: BoNT-A therapy should be considered for clinically relevant upper limb PSS that does not sufficiently respond to nonpharmacological treatment. In these cases, it should be entertained when the therapeutic goal is to support passive functions (prevention of contractures; hygiene, washing, dressing) (level of evidence 1a, quality of evidence moderate, $\mathrm{B}+$ ) and can be used in selected cases to support active function (level of evidence 1a, quality of evidence low, 0 ).

\subsubsection{Treatment Outcomes: Lower Limbs}

In chronic ankle flexion spasticity with spastic pes equines and equinovarus gait pattern, significant reduction of muscle tone in the ankle following treatment with abobotulinumtoxinA and onabotulinumtoxin $\mathrm{A}$ by intramuscular injections into the calf muscle could be demonstrated after stroke (Pittock et al. 2003; Kaji et al. 2010; Wein et al. 2018) (level of evidence 1b) and after stroke and TBI (Gracies et al. 2017) (level of evidence 1b). Additionally a reduced use of gait tools (orthosis) and an improvement of the Clinical Global Impression Scale in gait were shown ((Pittock et al. 2003; Gracies et al. 2017; Wein et al. 2018), level of evidence 1b). However, no significant improvements in longitudinal gait parameters (e.g., gait speed and gait endurance) of the patients treated with BoNT-A could be achieved in that trials.

One systematic review (mainly by observational studies and case series, 14 studies, 181 patients) also shows a reduction of clonus activity in patients with chronic ankle flexion spasticity as an improvement in a different positive sign of the UMNS that velocity-dependent increase in muscle tone from BoNT treatment (Thanikachalam et al. 2017) (level of evidence 3a).

With respect to the treatment of hip and knee spasticity using BoNT-A injections (abobotulinumtoxinA, onabotulinumtoxinA, and incobotulinumtoxinA), clinical studies were able to show reduction of spastic movement disorders in hip and knee in the chronic stage of spasticity of different etiologies (stroke, traumatic brain injury, multiple sclerosis, and others), and it was also possible to conclude that interventions with BoNT-A showed improving mobility ((Hyman et al. 2000; Rosales and Chua-Yap 2008; Wissel et al. 2017), level of evidence 1b).

In an open dose-escalation study with incobotulinumtoxinA, dose escalation from 400 units to 600 units and 800 units showed increasing numbers of treated upper and lower limb muscles or spastic patterns (combinations of typical spastic muscle activation, e.g., spastic pes equines, pes varus, flexed elbow or wrist, and spastic fist) per escalation level and led to almost double the number of patients, who were able to walk independently without increasing the incidence of side effects at higher dose levels (Wissel et al. 2017) (level of evidence 2b).

Recommendations: BoNT-A therapy can be considered for clinically relevant lower limb PSS (ankle, knee, or hip) that does not sufficiently respond to nonpharmacological treatment (level of evidence 1b, quality of evidence moderate, 0 (functional benefit uncertain)). It is also an option to treat functionally relevant sustained clonus (level of evidence $3 \mathrm{a}$, quality of evidence low, 0 ). 


\subsubsection{Treatment Outcomes: Spasticity- or Spasm-Associated Pain}

Neuropathic (e.g., spontaneous burning pain in plegic limbs), nociceptive (e.g., knee joint pain when starting walking), spasm-related, and spasticity-associated (stretch- or exercise-induced muscle pain) pain following stroke are all part of the decision-making process with respect to selection of symptomatic treatment and treatments should be tailored accordingly (Finnerup 2017). Reduction of spasmrelated and stretch- or exercise-induced spasticity-associated pain in spastic limb segments after injections of upper and lower extremity onabotulinumtoxinA in chronic spasticity was observed in a cohort study with 60 patients with mixed etiologies of spasticity in an open-label observational study and a randomized, placebo-controlled study in 273 patients following stroke and TBI ((Wissel et al. 2000, 2016), level of evidence $2 b$ and 1b). Spasticity-associated stretch- or exerciseinduced arm (Fheodoroff et al. 2017) or shoulder pain (Yelnik et al. 2007; Lim et al. 2008) also showed favorable influence by injections of abobotulinumtoxinA, incobotulinumtoxinA, and onabotulinumtoxinA (Fheodoroff et al. 2017, level of evidence 2b; Yelnik et al. 2007; Lim et al. 2008, level of evidence 1b).

Recommendations: BoNT-A therapy can be considered to treat spasm-related and stretch- or exercise-induced spasticity-associated pain in spastic limb segments, both in the upper or lower extremity (level of evidence 1b, quality of evidence low [partially indirect], 0).

\subsubsection{Botulinum Toxin A Injection Guidance}

It is believed that in order to improve the accuracy of BoNT injection, instrumented guidance using ultrasonography (US), electrical stimulation (ES), and electromyography (EMG) may be superior to a non-guided (e.g., pure anatomic localization) technique. Both ES and US appear to yield superior results over non-guided injection technique when BoNT is injected in wrist and finger flexors (Picelli et al. 2014; Santamato et al. 2014). ES (Chin et al. 2005; Picelli et al. 2012) and US (Picelli et al. 2012) have also been shown to be superior to anatomical guidance and EMG when injecting the triceps surae. A systematic review indicated that instrumented injection guidance is more effective than manual needle placement and showed similar effectiveness of US and ES for upper and lower limb spasticity in stroke (Grigoriu et al. 2015). A more recent investigation did not find a significant difference in clinical results among the various injection techniques when analyzing results of BoNT injections in a setting of an outpatient clinic (Zeuner et al. 2017). Thus, there is still no clear evidence that one injection technique is superior over another, but current literature suggests that for certain muscles instrument-guided injection is superior to pure anatomic localization (deep neck, forearm, and deep calf muscles).

Recommendation: Both US, ES, and EMG guidance can be used and are especially relevant when smaller or deeper muscles are injected (level of evidence 2a, quality of evidence low, 0). For the injection of larger superficial muscles, noninstrumented manual needle placement can be adequate (level of evidence 2a, quality of evidence low, 0 ). 


\subsubsection{BoNT-A Products}

BoNT-A drugs available are different and not interchangeable. (BoNT-B is also commercially available, but will not be included in this review considering its limited clinical use and scarcity of studies in spasticity. While BoNT-C and BoNT-F have been tried in human studies are up to now exclusive to dystonia.) The currently available BoNT-A drugs in Europe and North America are: abobotulinumtoxinA (Dysport $^{\circledR}$; Ipsen Ltd., Slough, Berks, UK), incobotulinumtoxinA (Xeomin ${ }^{\circledR}$; Merz Pharmaceuticals, Frankfurt/M, Germany), and onabotulinumtoxinA (Botox ${ }^{\circledR}$; Allergan Inc., Irvine, CA, USA). A recent entry in the market, daxibotulinumtoxinA (Revance, USA) is currently being investigated in both dystonia and spasticity. Additional BoNT-A drugs used in other countries (South America, India, and Asia) to manage spastic hypertonia and movement disorders include the Chinese BoNT-A Hengli $^{\circledR}$ (Lanzhou Institute of Biological Products, Lanzhou, Gansu Province, China) and marketed as Prosigne ${ }^{\circledR}$ elsewhere (e.g., Brazil). Neuronox ${ }^{\circledR}$ (Medy-Tox, Ochang, South Korea) is another BoNT-A available in some Asian countries. It is important to note that there has been no published systematic head-to-head comparison of the clinical properties, safety, and efficacy of commercially available formulations of BoNT-A. Hence, clinicians choose a BoNT-A formulation to treat PSS based on availability and experience with particular formulations, and no recommendation can be made regarding choice of a BoNT-A formulation in PSS.

\subsubsection{BoNT-A Dosing}

Appropriate dosing of BoNT-A is crucial in optimizing treatment outcomes and mitigating dose-related adverse events. Unfortunately, there is scarcity of doseranging studies (Simpson et al. 1996; Hyman et al. 2000; Gracies et al. 2015, 2017; Wissel et al. 2017) to allow evidence-based clinical decision-making with regard to dose selection for specific muscles. Hence, clinicians choose doses based on drugspecific summaries of product characteristic in package inserts, clinical experience, availability of toxins, and expert consensus recommendations ((Wissel et al. 2009; Esquenazi et al. 2013; Schramm et al. 2014), level of evidence 3).

Physician surveys suggest that many believe that greater flexibility in dosing might benefit some patients (Bensmail et al. 2014; Wissel 2018). Recently, a prospective, open-label, single-arm, multicenter, dose-titration study using high doses of incobotulinumtoxinA (up to $800 \mathrm{U}$ ) did not yield new safety signals (Wissel et al. 2017) (level of evidence 2b). In this particular trial, it was shown that high doses of incobotulinumtoxinA enabled simultaneous treatment of upper and lower limb and was associated with higher treatment goal attainment and no increase in side effect rate.

\subsubsection{Adjuvant Therapies to BoNT-A}

In a systematic review (without meta-analysis) of nine randomized trials (7 with neuromuscular electrostimulation, 2 with functional electrostimulation, 182 participants), it was shown that adjuvant electrostimulation therapy enhances the effect of BoNT-A injections on spasticity (Intiso et al. 2017) (level of evidence 1a). In two of these studies (both RCTs), spasticity was more responsive to additional 
electrostimulation after BoNT-A treatment than to additional stretching, but showed comparable effects as taping and lower effects than additional shock wave therapy. Neuromuscular electrostimulation was mostly performed in the studies for $30 \mathrm{~min}$, 1 to 6 times a day for 3 to 5 days after the injection. In another systematic review, 17 studies were included; it was shown that the effect of BoNT-A injections could be improved by various adjuvant therapies (Mills et al. 2016). Thus, an added benefit in the sense of a stronger tonus reduction (MAS $\geq 1$ ) was demonstrated to be proven for concomitant physiotherapy, modified constraint-induced movement therapy (mCIMT), electrostimulation, casting and dynamic splint treatment compared to BoNT-A alone, but not for taping, segmental muscle vibration, cyclic functional electrostimulation, or motorized arm ergometer (Mills et al. 2016) (level of evidence 1a).

Individual examples from selected studies are: After BoNT-A treatment of spastic calf muscles, a 1-week casting and to a lesser extent a 1-week taping showed better results with regard to spasticity reduction, increase of passive range of motion, and increased walking distance per time ( 6 min walking test) for 3 months after inclusion compared to a 1-week manual stretching by a physiotherapist following injection of BoNT-A (Carda et al. 2011) (level of evidence 1b). In another randomized controlled study for spastic pes equinus, no relevant additional effect of taping could be demonstrated (Karadag-Saygi et al. 2010) (level of evidence 1b).

In a Cochrane review, limited evidence could be provided as to whether and how multi-professional rehabilitation after BoNT-A treatment could improve spasticity in patients following stroke (3 RCS with 91 participants after stroke; (Demetrios et al. 2013), level of evidence 1a). With low-quality studies (GRADE) for mCIMT treatment following BoNT-A treatment, an improvement in active motor function and spasticity reduction could be shown. With very low-quality studies (GRADE) for occupational therapy following BoNT-A treatment improved in elbow mobility using a dynamic elbow extension splint compared to ergotherapy alone could be shown.

Recommendations: Neuromuscular electrostimulation applied for 3 to 5 days after BoNT-A therapy can be considered to enhance treatment effects in treated muscle groups (level of evidence 1a, quality of evidence low (risk of bias), 0). Safety aspects for the medical products used need to be taken into account. Other adjuvant therapies such as casting taping, mCIMT, and dynamic splint treatment can be used as individually indicated (level of evidence 1a, quality of evidence very low (risk of bias, inconsistency), 0). Active motor training and robotic training should be tailored to individual goals (refer to chapters on arm rehabilitation and mobility).

\subsection{Neurolysis}

Nerve blocks with neurolytic agents (phenol and alcohol) are effective in managing focal spasticity (Petrillo and Knoploch 1988; Chua and Kong 2000; Karri et al. 2017). For neurolysis, concentration of phenol usually ranges for $5 \%$ to $7 \%$, while 
concentration of alcohol varies from $50 \%$ to $100 \%$. These agents denature proteins in axons and membranes nonselectively in both afferent and efferent nerve fibers, leading to denervation and degeneration of spindles (Bodine-Fowler et al. 1996). Therefore, it requires precise localization and injection of these agents to the nerve fibers at the trunk, branch, or motor points of the target nerves. Precision localization is usually achieved with guidance of ultrasound imaging and/or electrical stimulation (Karri et al. 2017). Neurolysis usually produce immediate anesthesic effects and a later neurolytic effect on spasticity reduction. The duration depends on the dose, accuracy of injection, and repeated injections, ranging from 3 to 9 months. Unlike BoNT-A injections, nerve blocks can be repeated as early as several days. Common adverse effects include post-injection dysesthesia, localized swelling, and excessive weakness. In a recent retrospective chart review of 293 procedures, phenol neurolysis has a relatively favorable safety profile, including pain (4.0\%), swelling and inflammation $(2.7 \%)$, dysesthesia $(0.7 \%)$, and hypotension $(0.7 \%)$ (Karri et al. 2017). Although nerve blocks are widely used to manage spasticity, there is paucity of evidence of efficacy and safety based on randomized controlled studies. In a randomized, double-blind trial which compared phenol neurolysis and BoNT-A injection in the treatment of ankle and foot spasticity after stroke (Kirazli et al. 1998), the authors reported that both interventions were effective in plantar flexor spasticity reduction. The benefits were more significant in the BoNT-A group at weeks 2 and 4 post-injection, while there was no significant difference between two interventions at weeks 8 and 12 .

Recommendations: Phenol and alcohol neurolysis can be considered for clinically relevant PSS that does not sufficiently respond to nonpharmacological treatment (and oral medication), especially when BoNT-A treatment is not feasible (level of evidence $2 \mathrm{~b}$, quality of evidence low, 0 ). The possibility of long-term unwanted side effects, especially neuropathic pain following mixed nerve injections, need to be taken into account.

\subsection{Intrathecal Baclofen (ITB)}

Intrathecal baclofen therapy (ITB) is effective in managing post-stoke spastic hypertonia (Meythaler et al. 2001; Ivanhoe et al. 2006). It is licensed in the European Union to manage severe chronic spasticity in children between ages 4 and 18 years that is recalcitrant to oral spasmolytics and for managing severe spasticity of both cerebral and spinal etiology. In the United States (US), ITB is approved by the US Food and Drug Administration (US FDA) for managing severe spasticity of both cerebral and spinal etiology. Although few randomized, controlled trials of safety and efficacy exist, ITB has high levels of satisfaction among users of 10-24 years' duration (Mathur et al. 2014).

The US FDA recommends initiation of ITB therapy 1-year after disease onset, but a consensus panel of ITB experts suggested that ITB can be considered as early as 3-6 months post-stroke when spasticity is not controlled by other modalities or a patient is unable to tolerate side effects of other treatments (Francisco et al. 2006). 
A more recent consensus panel of experts (Saulino et al. 2016) recommended early consideration of ITB to avoid or delay various complications of spasticity and mitigate subsequent functional impairments. The same group commented that ITB should be considered only if other treatments have failed.

Meythaler et al. (2001) demonstrated superiority of ITB over placebo in a small cohort of stroke patients. Twenty-one stroke survivors with severe spasticity received either baclofen or placebo intrathecally. At $6 \mathrm{~h}$ post-bolus infusion, the average (mean $\pm \mathrm{SD}$ ) paretic lower limb Ashworth scores of the group that received baclofen decreased significantly from $3.3 \pm 1.2$ to $1.4 \pm 0.7(P<0.0001)$, spasm score from $1.2 \pm 1.2$ to $0.1 \pm 0.3(P=0.0224)$, and reflex score from $2.1 \pm 1.2$ to $0.1 \pm 0.5$ $(P<0.0001)$, compared to the group that received placebo. Seventeen subjects then received an intrathecal pump for continuous baclofen infusion. At 12 months following implantation and with an average daily dose of 268 micrograms per day, the average lower limb Ashworth score of the paretic side decreased from $3.7 \pm 1.0$ to $1.8 \pm 1.1(P<0.0001)$, the spasm score decreased from $1.2 \pm 1.3$ to $0.6 \pm 1.0$ $(P=0.4282)$, and the reflex score decreased from $2.4 \pm 1.3$ to $1.0 \pm 1.3(P<0.0001)$.

The effect of ITB on ambulant stroke survivors is controversial. Small reports (Francisco and Boake 2003; Remy-Neris et al. 2003) showed improvement in gait speed following ITB therapy, but others demonstrated otherwise (Kofler et al. 2009).

A recent randomized, controlled, open-label, multicenter trial also demonstrated superiority of ITB therapy over conventional medical management (cMM) with oral spasmolytics in terms of efficacy (mean Ashworth scale score reduction, -0.99 (ITB) vs. -0.43 (cMM); Hodges-Lehmann estimate -0.667 (95.1\% CI -1.0000 to $-0.1667) ; p=0.0140$ ) and pain control (numbers). While more subjects who received ITB reported adverse events (24/25 patients, 96\%; 149 events) compared to those who received cMM (22/35, 63\%; 77 events), no new safety signals were discovered (Creamer et al. 2018a, b).

Recommendations: ITB can be considered for clinically relevant severe segmental or generalized PSS that does not sufficiently respond to other interventions (level of evidence 1b, quality of evidence moderate, 0 (benefit risk for harm assessment)). ITB treatment ought to be tested, initiated, adjusted, and monitored with long-term support (including emergency work-up when indicated) by physicians experienced with the treatment (level of evidence 1b, quality of evidence moderate, $\mathrm{A}+$ (benefit risk for harm assessment)).

\subsection{Surgical Management}

Surgical procedures at the spinal cord level, such as interventions in the posterior root entry zone or dorsal rhizotomy, may be introduced in severe cases of spasticity after stroke, which are otherwise untreatable and represent a possibility of avoiding abnormal positions due to a severe spasticity and its complications in care, hygiene, pain, and contractures (review by (Chambers 1997), level of evidence 3a). One positive case report with persistent effects for the partial posterior rhizotomy is available in the literature ((Fukuhara and Kamata 2004), level of evidence 4). Controlled 
studies on these procedures in children with cerebral palsy are published, and studies in adults following stroke are not available. One major criticism with this surgical procedure is that it may only bring temporal improvements.

Partial neurotomy of the motor branches of the tibial nerve to the triceps surae muscle is an established neurosurgical procedure and leads to reduced spastic muscle tone in pes equinus or equinovarus deformity, and therefore can improve positioning of the foot and can reduce associated pain during walking ((Sindou and Mertens 2000), level of evidence 2b).

Following reports of successful orthopedic surgery with fasciotomy, transfer of muscle attachments, tendon transfer, and even bony surgery to increase upper and lower limb function in persons with low functioning spastic upper and lower limbs following spinal cord injury (Fox et al. 2018) (level of evidence 3) in the last 10 years also in chronic stroke patients with limited upper and lower limb function, those procedures became more available, but no large cohort studies are published. Fascia, tendon and muscle lengthening (e.g., Achilles tendon lengthening, soleus muscle fasciotomy, or lengthening), and tendon transfer surgery (e.g., transfer of the tendon of the flexor carpi ulnaris muscle to the dorsal aspect of the wrist or tibialis posterior split transfer and transpositioning of a part of the tendon to the dorsal aspect of the foot) may help correcting spastic hand or foot posture and help to improve active wrist and foot extension to improve residual function as well as preventing complications due to spastic posturing and development of contracture ((Duquette and Adkinson 2018), level of evidence 4; (Sturbois-Nachef et al. 2019), level of evidence 3). Despite the recognized effectiveness of orthopedic surgery for neuro-orthopedic disorders like chronic stroke patients with spastic contractures, few studies have formally evaluated them. Hence, there is a need for research to provide evidence to support orthopedic surgery for treating such neuro-orthopedic disorders (Genet et al. 2018).

Thumb-in-palm deformity is a well-known cause of disability in the chronic stroke population with upper limb spasticity but no reports on surgical treatments are published. On the other hand, surgical correction of spastic flexion of the thumb due to in juvenile cerebral palsy is established and it was evaluated in a Cochrane review. The authors describe a positive effect noted by patients (patient-related outcome) and the surgeons. The authors of the review critiqued that no standardized surgical procedure is defined, and different assessment methods in evaluation of the results following surgery were used. The authors concluded that based on the data available an evidence-based assessment of this treatment, procedure was not possible (Smeulders et al. 2005) (level of evidence 1a).

Recommendations: In individual cases, after careful examination in the multiprofessional team and exhaustion of other reversible treatment options for spastic movement disorder, surgical procedures may be considered as treatment option in chronic spastic movement disorder following stroke (level of evidence 4, quality of evidence very low, 0 ). 


\section{References}

Ansari NN, Naghdi S, Hasson S, Azarsa MH, Azarnia S (2008) The modified Tardieu scale for the measurement of elbow flexor spasticity in adult patients with hemiplegia. Brain Inj 22:1007-1012

Ansari NN, Naghdi S, Hasson S, Rastgoo M, Amini M, Forogh B (2013) Clinical assessment of ankle plantarflexor spasticity in adult patients after stroke: inter-and intra-rater reliability of the modified Tardieu scale. Brain Inj 27:605-612

Ashford S, Fheodoroff K, Jacinto J, Turner-Stokes L (2016) Common goal areas in the treatment of upper limb spasticity: a multicentre analysis. Clin Rehabil 30:617-622

Baker JA, Pereira G (2015) The efficacy of Botulinum toxin a on improving ease of care in the upper and lower limbs: a systematic review and meta-analysis using the grades of recommendation, assessment, development and evaluation approach. Clin Rehabil 29:731-740

Baker JA, Pereira G (2016) The efficacy of Botulinum toxin a for limb spasticity on improving activity restriction and quality of life: a systematic review and meta-analysis using the GRADE approach. Clin Rehabil 30:549-558

Banky M, Clark RA, Mentiplay BF, Olver JH, Kahn MB, Williams G (2019) Toward accurate clinical spasticity assessment: validation of movement speed and joint angle assessments using smartphones and camera tracking. Arch Phys Med Rehabil 100(8):1482-1491

Bensmail D, Hanschmann A, Wissel J (2014) Satisfaction with botulinum toxin treatment in poststroke spasticity: results from two cross-sectional surveys (patients and physicians). J Med Econ 17:618-625

Bodine-Fowler SC, Allsing S, Botte MJ (1996) Time course of muscle atrophy and recovery following a phenol-induced nerve block. Muscle Nerve 19:497-504

Brashear A, Gordon MF, Elovic E et al (2002a) Intramuscular injection of botulinum toxin for the treatment of wrist and finger spasticity after a stroke. N Engl J Med 347:395-400

Brashear A, Zafonte R, Corcoran M et al (2002b) Inter- and intrarater reliability of the Ashworth scale and the disability assessment scale in patients with upper-limb poststroke spasticity. Arch Phys Med Rehabil 83:1349-1354

Calota A, Feldman AG, Levin MF (2008) Spasticity measurement based on tonic stretch reflex threshold in stroke using a portable device. Clin Neurophysiol 119:2329-2337

Carda S, Invernizzi M, Baricich A, Cisari C (2011) Casting, taping or stretching after botulinum toxin type a for spastic equinus foot: a single-blind randomized trial on adult stroke patients. Clin Rehabil 25:1119-1127

CEBM (2009) Oxford center for evidence-based medicine-levels of evidence. In: Last version from March 2009. https://www.cebm.net/2009/06/oxford-centre-evidence-based-medicinelevels-evidence-march-2009/

Chambers HG (1997) The surgical treatment of spasticity. Muscle Nerve Suppl 6:S121-S128

Chin TY, Nattrass GR, Selber P, Graham HK (2005) Accuracy of intramuscular injection of botulinum toxin a in juvenile cerebral palsy: a comparison between manual needle placement and placement guided by electrical stimulation. J Pediatr Orthop 25:286-291

Chua KS, Kong KH (2000) Alcohol neurolysis of the sciatic nerve in the treatment of hemiplegic knee flexor spasticity: clinical outcomes. Arch Phys Med Rehabil 81:1432-1435

Creamer M, Cloud G, Kossmehl P et al (2018a) Intrathecal baclofen therapy versus conventional medical management for severe poststroke spasticity: results from a multicentre, randomised, controlled, open-label trial (SISTERS). J Neurol Neurosurg Psychiatry 89:642-650

Creamer M, Cloud G, Kossmehl P et al (2018b) Effect of Intrathecal baclofen on pain and quality of life in poststroke spasticity. Stroke 49:2129-2137

Demetrios M, Khan F, Turner-Stokes L, Brand C, McSweeney S (2013) Multidisciplinary rehabilitation following botulinum toxin and other focal intramuscular treatment for post-stroke spasticity. Cochrane Database Syst Rev (6):CD009689 
Dong Y, Wu T, Hu X, Wang T (2017) Efficacy and safety of botulinum toxin type a for upper limb spasticity after stroke or traumatic brain injury: a systematic review with meta-analysis and trial sequential analysis. Eur J Phys Rehabil Med 53:256-267

Duquette SP, Adkinson JM (2018) Surgical management of spasticity of the forearm and wrist. Hand Clin 34:487-502

Esquenazi A, Albanese A, Chancellor MB et al (2013) Evidence-based review and assessment of botulinum neurotoxin for the treatment of adult spasticity in the upper motor neuron syndrome. Toxicon 67:115-128

Fheodoroff K, Kossmehl P, Wissel J (2017) Validity and reliability of the spasticity-associated arm pain scale. J Pain Manag Med 3:127

Fietzek UM, Kossmehl P, Schelosky L, Ebersbach G, Wissel J (2014) Early botulinum toxin treatment for spastic pes equinovarus - a randomized double-blind placebo-controlled study. Eur J Neurol 21:1089-1095

Finnerup NB (2017) Neuropathic pain and spasticity: intricate consequences of spinal cord injury. Spinal Cord 55:1046-1050

Flewellen EH, Nelson PE, Jones WP, Arens JF, Wagner DL (1983) Dantrolene dose-response in awake man: implications for management of malignant hyperthermia. Anesthesiology 59:275-280

Foley N, Pereira S, Salter K et al (2013) Treatment with botulinum toxin improves upperextremity function post stroke: a systematic review and meta-analysis. Arch Phys Med Rehabil 94:977-989

Fox IK, Miller AK, Curtin CM (2018) Nerve and tendon transfer surgery in cervical spinal cord injury: individualized choices to optimize function. Top Spinal Cord Inj Rehabil 24: 275-287

Francisco GE, Boake C (2003) Improvement in walking speed in poststroke spastic hemiplegia after intrathecal baclofen therapy: a preliminary study. Arch Phys Med Rehabil 84:1194-1199

Francisco GE, Li S (2015) Spasticity. In: Physical medicine and rehabilitation, 5th edn. Elsevier, Philadelphia

Francisco GE, McGuire JR (2012) Poststroke spasticity management. Stroke 43:3132-3136

Francisco GE, Yablon SA, Schiess MC, Wiggs L, Cavalier S, Grissom S (2006) Consensus panel guidelines for the use of intrathecal baclofen therapy in poststroke spastic hypertonia. Top Stroke Rehabil 13:74-85

Fukuhara T, Kamata I (2004) Selective posterior rhizotomy for painful spasticity in the lower limbs of hemiplegic patients after stroke: report of two cases. Neurosurgery 54:1268-1272. discussion $1272-1263$

Gao J, He W, Du LJ et al (2018) Quantitative ultrasound imaging to assess the biceps Brachii muscle in chronic post-stroke spasticity: preliminary observation. Ultrasound Med Biol 44(9): 1931-1940

Genet F, Denormandie P, Keenan MA (2018) Orthopaedic surgery for patients with central nervous system lesions: concepts and techniques. Ann Phys Rehabil Med 62(4):225-233

Gracies JM, Brashear A, Jech R et al (2015) Safety and efficacy of abobotulinumtoxinA for hemiparesis in adults with upper limb spasticity after stroke or traumatic brain injury: a double-blind randomised controlled trial. Lancet Neurol 14:992-1001

Gracies JM, Esquenazi A, Brashear A et al (2017) Efficacy and safety of abobotulinumtoxinA in spastic lower limb: randomized trial and extension. Neurology 89:2245-2253

Grigoriu AI, Dinomais M, Rémy-Néris O, Brochard S (2015) Impact of injection-guiding techniques on the effectiveness of Botulinum toxin for the treatment of focal spasticity and dystonia: a systematic review. Arch Phys Med Rehabil 96:2067-2078

Halpern R, Gillard P, Graham GD, Varon SF, Zorowitz RD (2013) Adherence associated with oral medications in the treatment of spasticity. PM R 5:747-756

Haugh A, Pandyan A, Johnson G (2006) A systematic review of the Tardieu scale for the measurement of spasticity. Disabil Rehabil 28:899-907 
Hesse S, Mach H, Frohlich S, Behrend S, Werner C, Melzer I (2012) An early botulinum toxin a treatment in subacute stroke patients may prevent a disabling finger flexor stiffness six months later: a randomized controlled trial. Clin Rehabil 26:237-245

Hyman N, Barnes M, Bhakta B et al (2000) Botulinum toxin (Dysport) treatment of hip adductor spasticity in multiple sclerosis: a prospective, randomised, double blind, placebo controlled, dose ranging study. J Neurol Neurosurg Psychiatry 68:707-712

Intiso D, Santamato A, Di Rienzo F (2017) Effect of electrical stimulation as an adjunct to botulinum toxin type a in the treatment of adult spasticity: a systematic review. Disabil Rehabil 39:2123-2133

Ivanhoe CB, Francisco GE, McGuire JR, Subramanian T, Grissom SP (2006) Intrathecal baclofen management of poststroke spastic hypertonia: implications for function and quality of life. Arch Phys Med Rehabil 87:1509-1515

Kaji R, Osako Y, Suyama K, Maeda T, Uechi Y, Iwasaki M (2010) Botulinum toxin type a in poststroke lower limb spasticity: a multicenter, double-blind, placebo-controlled trial. J Neurol 257:1330-1337

Kamper D, Harvey R, Suresh S, Rymer W (2003) Relative contributions of neural mechanisms versus muscle mechanics in promoting finger extension deficits following stroke. Muscle Nerve 28:309-318

Karadag-Saygi E, Cubukcu-Aydoseli K, Kablan N, Ofluoglu D (2010) The role of kinesiotaping combined with botulinum toxin to reduce plantar flexors spasticity after stroke. Top Stroke Rehabil 17:318-322

Karri J, Mas MF, Francisco GE, Li S (2017) Practice patterns for spasticity management with phenol neurolysis. J Rehabil Med 49:482-488

Katrak PH, Cole AMD, Poulos CJ, McCauley JCK (1992) Objective assessment of spasticity, strength, and function with early exhibition of dantrolene sodium after cerebrovascular accident: a randomised double-blind controlled study. Arch Phys Med Rehabil 73:4-9

Kirazli Y, On AY, Kismali B, Aksit R (1998) Comparison of phenol block and botulinus toxin type $\mathrm{a}$ in the treatment of spastic foot after stroke: a randomized, double-blind trial. Am J Phys Med Rehabil 77:510-515

Kiresuk TJ, Sherman RE (1968) Goal attainment scaling: a general method for evaluating comprehensive community mental health programs. Community Ment Health J 4:443-453

Kofler M, Quirbach E, Schauer R, Singer M, Saltuari L (2009) Limitations of intrathecal baclofen for spastic hemiparesis following stroke. Neurorehabil Neural Repair 23:26-31

Krause T, Gerbershagen MU, Fiege M, Weisshorn R, Wappler F (2004) Dantrolene-a review of its pharmacology, therapeutic use and new developments. Anaesthesia 59:364-373

Lee KB, Hong BY, Kim JS et al (2019) Which brain lesions produce spasticity? An observational study on 45 stroke patients. PLoS One 14:e0210038

Levin MF, Feldman AG (1994) The role of stretch reflex threshold regulation in normal and impaired motor control. Brain Res 657:23-30

Li S, Kamper DG, Rymer WZ (2006) Effects of changing wrist positions on finger flexor hypertonia in stroke survivors. Muscle Nerve 33:183-190

Li F, Wu Y, Li X (2014) Test-retest reliability and inter-rater reliability of the modified Tardieu scale and the modified Ashworth scale in hemiplegic patients with stroke. Eur J Phys Rehabil Med 50:9-15

Lichtner V, Dowding D, Esterhuizen P, Closs SJ, Long AF, Corbett A, Briggs M (2014) Pain assessment for people with dementia: a systematic review of systematic reviews of pain assessment tools. BMC Geriatr 14:138

Lim JY, Koh JH, Paik NJ (2008) Intramuscular botulinum toxin-a reduces hemiplegic shoulder pain: a randomized, double-blind, comparative study versus intraarticular triamcinolone acetonide. Stroke 39:126-131

Lindsay C, Kouzouna A, Simcox C, Pandyan AD (2016) Pharmacological interventions other than botulinum toxin for spasticity after stroke. Cochrane Database Syst Rev 10:CD010362 
Malhotra S, Pandyan AD, Rosewilliam S, Roffe C, Hermens H (2011) Spasticity and contractures at the wrist after stroke: time course of development and their association with functional recovery of the upper limb. Clin Rehabil 25:184-191

Mathur SN, Chu SK, McCormick Z, Chang Chien GC, Marciniak CM (2014) Long-term intrathecal baclofen: outcomes after more than 10 years of treatment. PM R 6:506-513.e1

Medaer R, Hellebuyk H, Van Den Brande E, Saxena V, Thijs M, Kovacs L, Dehaen F (1991) Treatment of spasticity due to stroke: a double-blind, cross-over trial comparing baclofen with placebo. Acta Therapeut 17:323-331

Mehrholz J, Major Y, Meissner D, Sandi-Gahun S, Koch R, Pohl M (2005) The influence of contractures and variation in measurement stretching velocity on the reliability of the modified Ashworth scale in patients with severe brain injury. Clin Rehabil 19:63-72

Meseguer-Henarejos AB, SăNchez-Meca J, López-Pina JA, Carles-HernăNdez R (2018) Inter-and intra-rater reliability of the modified Ashworth scale: a systematic review and meta-analysis. Eur J Phys Rehabil Med 54:576-590

Meythaler JM, Guin-Renfroe S, Brunner RC, Hadley MN (2001) Intrathecal baclofen for spastic hypertonia from stroke. Stroke 32:2099-2109

Meythaler JM, Clayton W, Davis LK, Guin-Renfroe S, Brunner RC (2004) Orally delivered baclofen to control spastic hypertonia in acquired brain injury. J Head Trauma Rehabil 19:101-108

Mills PB, Finlayson H, Sudol M, O’Connor R (2016) Systematic review of adjunct therapies to improve outcomes following botulinum toxin injection for treatment of limb spasticity. Clin Rehabil 30:537-548

Montane E, Vallano A, Laporte JR (2004) Oral antispastic drugs in nonprogressive neurologic diseases: a systematic review. Neurology 63:1357-1363

Olvey EL, Armstrong EP, Grizzle AJ (2010) Contemporary pharmacologic treatments for spasticity of the upper limb after stroke: a systematic review. Clin Ther 32:2282-2303

Owens DK, Lohr KN, Atkins D et al (2010) AHRQ series paper 5: grading the strength of a body of evidence when comparing medical interventions-agency for healthcare research and quality and the effective health-care program. J Clin Epidemiol 63:513-523

Paulis WD, Horemans HL, Brouwer BS, Stam HJ (2011) Excellent test-retest and inter-rater reliability for Tardieu scale measurements with inertial sensors in elbow flexors of stroke patients. Gait Posture 33:185-189

Petrillo CR, Knoploch S (1988) Phenol block of the tibial nerve for spasticity: a long-term followup study. Int Disabil Stud 10:97-100

Picelli A, Bonetti P, Fontana C et al (2012) Accuracy of botulinum toxin type a injection into the gastrocnemius muscle of adults with spastic equinus: manual needle placement and electrical stimulation guidance compared using ultrasonography. J Rehabil Med 44:450-452

Picelli A, Lobba D, Midiri A, Prandi P, Melotti C, Baldessarelli S, Smania N (2014) Botulinum toxin injection into the forearm muscles for wrist and fingers spastic overactivity in adults with chronic stroke: a randomized controlled trial comparing three injection techniques. Clin Rehabil 28:232-242

Pittock SJ, Moore AP, Hardiman O et al (2003) A double-blind randomised placebo-controlled evaluation of three doses of botulinum toxin type a (Dysport) in the treatment of spastic equinovarus deformity after stroke. Cerebrovasc Dis 15:289-300

Platz T (2017) Practice guidelines in neurorehabilitation. Neurol Int Open 01:E148-E152

Platz T, Vuadens P, Eickhof C, Arnold P, Van Kaick S, Heise K (2008) REPAS, a summary rating scale for resistance to passive movement: item selection, reliability and validity. Disabil Rehabil 30:44-53

Platz T, Wissel J, Donauer E, Vogel M, Tholen R, Lehmler L (2019) S2k-Leitlinie: Therapie des spastischen Syndroms. DGNeurologie (online first/ahead of print). https://doi.org/10.1007/ s42451-019-0090-2 
Remy-Neris O, Denys P, Daniel O, Barbeau H, Bussel B (2003) Effect of intrathecal clonidine on group I and group II oligosynaptic excitation in paraplegics. Exp Brain Res 148:509-514

Rockwood K, Joyce B, Stolee P (1997) Use of goal attainment scaling in measuring clinically important change in cognitive rehabilitation patients. J Clin Epidemiol 50:581-588

Rosales RL, Chua-Yap AS (2008) Evidence-based systematic review on the efficacy and safety of botulinum toxin-a therapy in post-stroke spasticity. J Neural Transm (Vienna) 115:617-623

Rosales RL, Kong KH, Goh KJ et al (2012) Botulinum toxin injection for hypertonicity of the upper extremity within 12 weeks after stroke: a randomized controlled trial. Neurorehabil Neural Repair 26:812-821

Rosales RL, Efendy F, Teleg ES et al (2016) Botulinum toxin as early intervention for spasticity after stroke or non-progressive brain lesion: a meta-analysis. J Neurol Sci 371:6-14

Santamato A, Micello MF, Panza F et al (2014) Can botulinum toxin type a injection technique influence the clinical outcome of patients with post-stroke upper limb spasticity? A randomized controlled trial comparing manual needle placement and ultrasound-guided injection techniques. J Neurol Sci 347:39-43

Saulino M, Anderson DJ, Doble J, Farid R, Gul F, Konrad P, Boster AL (2016) Best practices for Intrathecal baclofen therapy: troubleshooting. Neuromodulation 19:632-641

Schramm A, Ndayisaba JP, Auf dem Brinke M et al (2014) Spasticity treatment with onabotulinumtoxin a: data from a prospective German real-life patient registry. J Neural Transm (Vienna) 121:521-530

Schünemann H, Brożek J, Guyatt G, Oxman A (2013) GRADE handbook for grading quality of evidence and strength of recommendations. The GRADE Working Group. Updated October 2013. www.guidelinedevelopment.org/handbook

Simpson DM, Alexander DN, O'Brien CF et al (1996) Botulinum toxin type a in the treatment of upper extremity spasticity: a randomized, double-blind, placebo-controlled trial. Neurology 46:1306-1310

Simpson DM, Gracies JM, Graham HK et al (2008) Assessment: Botulinum neurotoxin for the treatment of spasticity (an evidence-based review): report of the therapeutics and technology assessment Subcommittee of the American Academy of neurology. Neurology 70: $1691-1698$

Simpson DM, Gracies JM, Yablon SA, Barbano R, Brashear A (2009) Botulinum neurotoxin versus tizanidine in upper limb spasticity: a placebo-controlled study. J Neurol Neurosurg Psychiatry 80:380-385

Simpson DM, Hallett M, Ashman EJ et al (2016) Practice guideline update summary: Botulinum neurotoxin for the treatment of blepharospasm, cervical dystonia, adult spasticity, and headache report of the guideline development Subcommittee of the American Academy of neurology. Neurology 86:1818-1826

Sindou MP, Mertens P (2000) Neurosurgery for spasticity. Stereotact Funct Neurosurg 74:217-221

Singh P, Joshua AM, Ganeshan S, Suresh S (2011) Intra-rater reliability of the modified Tardieu scale to quantify spasticity in elbow flexors and ankle plantar flexors in adult stroke subjects. Ann Indian Acad Neurol 14:23-26

Sinkjaer T, Magnussen I (1994) Passive, intrinsic and reflex-mediated stiffness in the ankle extensors of hemiparetic patients. Brain 117:355-363

Smeulders M, Coester A, Kreulen M (2005) Surgical treatment for the thumb-in-palm deformity in patients with cerebral palsy. Cochrane Database Syst Rev (4):CD004093

Sommerfeld DK, Eek EU, Svensson AK, Holmqvist LW, von Arbin MH (2004) Spasticity after stroke: its occurrence and association with motor impairments and activity limitations. Stroke 35:134-139

Stamenova P, Koytchev R, Kuhn K, Hansen C, Horvath F, Ramm S, Pongratz D (2005) A randomized, double-blind, placebo-controlled study of the efficacy and safety of tolperisone in spasticity following cerebral stroke. Eur J Neurol 12:453-461 
Stevenson VL, Jarrett L (2006) Spasticity management: a practical multidisciplinary guide. Informa Healthcare, London

Sturbois-Nachef N, Allart E, Grauwin MY, Rousseaux M, Thevenon A, Fontaine C (2019) Tibialis posterior transfer for foot drop due to central causes: Long-term hindfoot alignment. Orthop Traumatol Surg Res 105:153-158

Thanikachalam V, Phadke CP, Ismail F, Boulias C (2017) Effect of Botulinum toxin on clonus: a systematic review. Arch Phys Med Rehabil 98:381-390

Turkel CC, Bowen B, Liu J, Brin MF (2006) Pooled analysis of the safety of botulinum toxin type a in the treatment of poststroke spasticity. Arch Phys Med Rehabil 87:786-792

Turner-Stokes L, Baguley IJ, De Graaff S, Katrak P, Davies L, McCrory P, Hughes A (2010) Goal attainment scaling in the evaluation of treatment of upper limb spasticity with botulinum toxin: a secondary analysis from a double-blind placebo-controlled randomized clinical trial. J Rehabil Med 42:81-89

Turner-Stokes L, Rose H, Ashford S, Singer B (2015) Patient engagement and satisfaction with goal planning: impact on outcome from rehabilitation. Int J Ther Rehabil 22:210-216

Tyson SF, Brown P (2014) How to measure pain in neurological conditions? A systematic review of psychometric properties and clinical utility of measurement tools. Clin Rehabil 28:669-686

van Kuijk AA, Geurts AC, Bevaart BJ, van Limbeek J (2002) Treatment of upper extremity spasticity in stroke patients by focal neuronal or neuromuscular blockade: a systematic review of the literature. J Rehabil Med 34:51-61

Watkins CL, Leathley MJ, Gregson JM, Moore AP, Smith TL, Sharma AK (2002) Prevalence of spasticity post stroke. Clin Rehabil 16:515-522

Wein T, Esquenazi A, Jost WH, Ward AB, Pan G, Dimitrova R (2018) OnabotulinumtoxinA for the treatment of Poststroke distal lower limb spasticity: a randomized trial. PM R 10:693-703

Whiting PF, Wolff RF, Deshpande S et al (2015) Cannabinoids for medical use: a systematic review and meta-analysis. JAMA 313:2456-2473

Winstein CJ, Stein J, Arena R et al (2016) Guidelines for adult stroke rehabilitation and recovery: a guideline for healthcare professionals from the American Heart Association/American Stroke Association. Stroke 47:e98-e169

Winter T, Wissel J (2013) Behandlung der Spastizität nach Schlaganfall. Konsultationsfassung der DGNR-Leitlinie. Neurol Rehabil 19:285-309

Wissel J (2018) Towards flexible and tailored botulinum neurotoxin dosing regimens for focal dystonia and spasticity-insights from recent studies. Toxicon 147:100-106

Wissel J, Muller J, Dressnandt J, Heinen F, Naumann M, Topka H, Poewe W (2000) Management of spasticity associated pain with botulinum toxin a. J Pain Symptom Manage 20:44-49

Wissel J, Haydn T, Muller J, Brenneis C, Berger T, Poewe W, Schelosky LD (2006) Low dose treatment with the synthetic cannabinoid Nabilone significantly reduces spasticity-related pain: a double-blind placebo-controlled cross-over trial. J Neurol 253:1337-1341

Wissel J, Ward AB, Erztgaard P et al (2009) European consensus table on the use of botulinum toxin type a in adult spasticity. J Rehabil Med 41:13-25

Wissel J, Schelosky LD, Scott J, Christe W, Faiss JH, Mueller J (2010) Early development of spasticity following stroke: a prospective, observational trial. J Neurol 257:1067-1072

Wissel J, Verrier M, Simpson DM, Charles D, Guinto P, Papapetropoulos S, Sunnerhagen KS (2015) Post-stroke spasticity: predictors of early development and considerations for therapeutic intervention. PM R 7:60-67

Wissel J, Ganapathy V, Ward AB et al (2016) OnabotulinumtoxinA improves pain in patients with post-stroke spasticity: findings from a randomized, double-blind, placebo-controlled trial. J Pain Symptom Manage 52:17-26

Wissel J, Bensmail D, Ferreira JJ et al (2017) Safety and efficacy of incobotulinumtoxinA doses up to $800 \mathrm{U}$ in limb spasticity: the TOWER study. Neurology 88:1321-1328 
Wu CH, Ho YC, Hsiao MY, Chen WS, Wang TG (2017) Evaluation of post-stroke spastic muscle stiffness using shear wave ultrasound elastography. Ultrasound Med Biol 43:1105-1111

Yelnik AP, Colle FM, Bonan IV, Vicaut E (2007) Treatment of shoulder pain in spastic hemiplegia by reducing spasticity of the subscapular muscle: a randomised, double blind, placebocontrolled study of botulinum toxin A. J Neurol Neurosurg Psychiatry 78:845-848

Zeuner KE, Knutzen A, Kuhl C et al (2017) Functional impact of different muscle localization techniques for Botulinum neurotoxin a injections in clinical routine management of post-stroke spasticity. Brain Inj 31:75-82

Open Access This chapter is licensed under the terms of the Creative Commons AttributionNonCommercial-NoDerivatives 4.0 International License (http://creativecommons.org/ licenses/by-nc-nd/4.0/), which permits any noncommercial use, sharing, distribution and reproduction in any medium or format, as long as you give appropriate credit to the original author(s) and the source, provide a link to the Creative Commons license and indicate if you modified the licensed material. You do not have permission under this license to share adapted material derived from this chapter or parts of it.

The images or other third party material in this chapter are included in the chapter's Creative Commons license, unless indicated otherwise in a credit line to the material. If material is not included in the chapter's Creative Commons license and your intended use is not permitted by statutory regulation or exceeds the permitted use, you will need to obtain permission directly from the copyright holder.

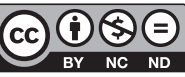

